\title{
ACUTE HAEMATOGENOUS OSTEOMYELITIS WITH SPECIAL REFERENCE TO OSTEITIS OF THE NECK OF THE FEMUR*
}

\author{
BY \\ J. H. LOUW and B. SHANDLING \\ From the Department of Surgery, University of Cape Town
}

Acute haematogenous osteomyelitis is still a common disease in Cape Town. During the past eight years we have treated 331 cases ( 351 bones) in the University of Cape Town teaching hospitals; $85 \%$ of these were children of 12 years and under, the disease accounting for $3 \cdot 3 \%$ of the admissions to our Children's hospital where we have set aside a special ward for patients suffering from osteomyelitis.

Not only is the disease common but it seems to be increasing in frequency and in severity. Thus there has been a steady increase in the annual admissions (Fig. 1) and, whereas in 1949 only $24 \%$ of our cases were regarded as severe (Du Plessis, 1953), in 1959 the proportion had increased to $57 \%$ (Shandling, 1960).

Our cases have recently been reviewed by one of us (Shandling, 1960), and our findings in regard to age and sex incidence, social status, pre-existing septic foci and predisposing trauma are in keeping with those of others (Altemeier and Helmsworth, 1945; Agerholm and Trueta, 1946; Caldwell and Wickstrom, 1950; White and Dennison, 1952; Dennison, 1952; Trueta and Morgan, 1954). The bones most commonly affected were upper tibiae, lower femora and tibiae and upper femoral shafts (Fig. 2). In this paper we wish to discuss osteomyelitis of the neck of the femur of which we have had 27 cases ( 28 bones) since 1952. Before doing so, a consideration of the racial incidence of osteomyelitis may be of interest.

\section{Racial Incidence}

The population of Cape Town (Fig. 3) consists of $41 \%(310,000)$ Whites of European origin, $45 \%(340,000)$ Coloureds (mixture of original South African Yellow races, Whites and Asiatics) and $14 \%(107,000)$ Bantu (Negroids). As far as

* A paper read at a meeting of the British Association of Paediatric Surgeons held in London in July 1960. osteitis is concerned, we found $16 \%$ (53) in Whites, $80 \%$ (267) in Coloureds and 4\% (11) in Bantu. This might suggest a racial predisposition as far as the Coloureds are concerned, but it is in fact a social and economic problem. It has long been noted that osteomyelitis is a disease of the poor (Mitchell, 1928), and Stevenson (1946) has pointed out that the disease is rare in private practice. In Cape Town, osteomyelitis is rare in the wealthier private patients and practically all our Coloured hospital patients come from very poor homes where lack of cleanliness and undernutrition are rife. On the other hand the Bantu children live in similar, often worse, circumstances and yet the disease is rare among them.

\section{Osteomyelitis of the Neck of the Femur}

Our 27 cases of osteomyelitis of the neck of the femur have followed the usual pattern. All the

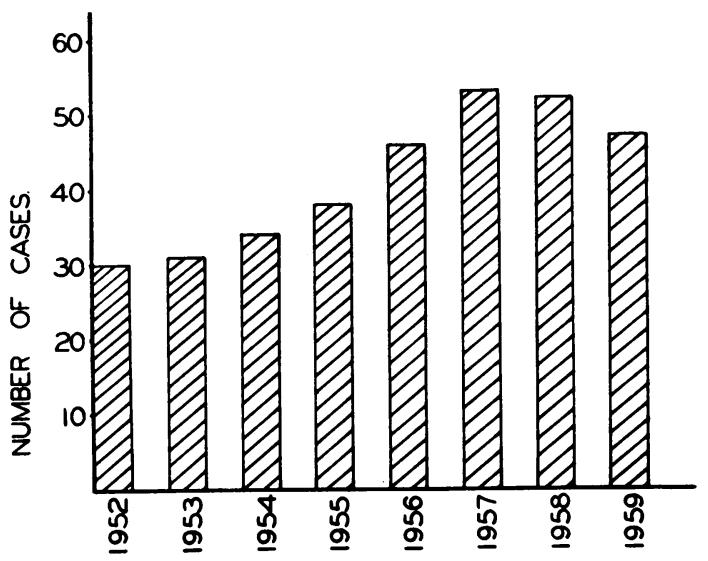

$0.007 \%$ OF POPULATION.

85\% UNDER 12 YEARS - $3.3 \%$ OF ADMISSIONS TO CHILDREN'S HOSPITAL.

FIG. 1.-Annual figures of acute haematogenous osteomyelitis from University of Cape Town series 1952-1959-331 patients. 


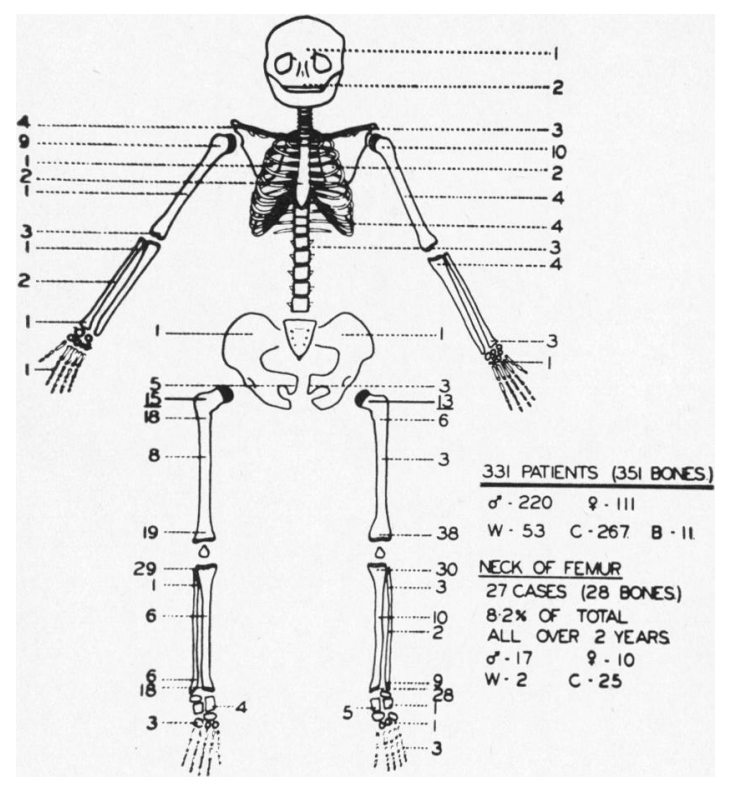

FIG. 2.-Diagram of bones involved in a series of 331 cases of acute osteomyelitis.

CAPE TOWN POPULATION $-757,000$.

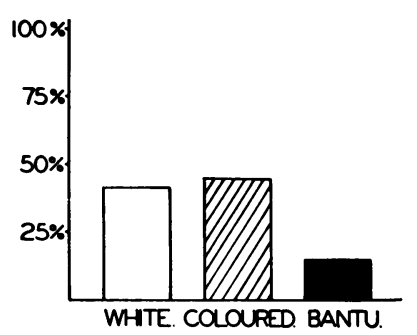

ACUTE OSTEOMYELITIS - 331.

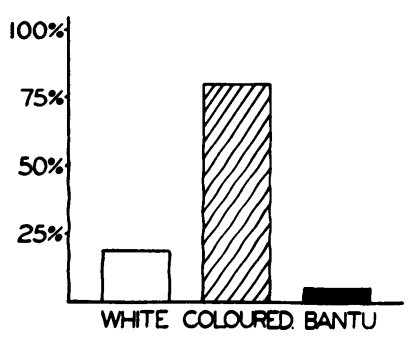

FIG. 3.-Racial incidence. patients were over 2 years of age (comprising $9 \%$ of our cases of osteomyelitis in patients over 2 years), their ages ranging from 3 to 14 years with the highest incidence in the 10- to 12-year-olds (12 cases). Twenty-five were Coloured and two were White (no Bantu); there were 17 males and 10 females. It has often been pointed out that osteomyelitis in infants under 2 years differs from the disease in older children (Ober, 1938; Trueta and Morgan, 1954) and we wish to make it clear that the following remarks apply only to the older age group.

Bacteriology. The infecting organism was cultured from the patients' blood and/or pus in 24 cases and was found to be the Micrococcus pyogenes aureus in all (Table 1). In $16(66 \%)$ it was sensitive to all the antibiotics including penicillin, in seven it was sensitive to streptomycin, chloramphenicol, tetracycline, erythromycin and novobiocin, but not to penicillin, and in one to chloramphenicol and erythromycin only. (In our cases in general the Mic. pyogenes aureus was the responsible organism in $98 \%$; it was sensitive to all the antibiotics including penicillin in $82 \%$, to streptomycin, chloramphenicol, erythromycin, tetracycline and novobiocin, but not penicillin in $14 \%$ and to chloramphenicol and erythromycin only in $4 \%$.)

Clinical Presentation. The clinico-pathological features are conveniently divided into three groups (Table 2) depending upon the general condition of the patient on admission to hospital: (1) Bacteraemia and inflammatory fever; (2) septicaemia, or (3) severe toxaemia.

(1) Bacteraemia and Inflammatory Fever11 CASES. The general condition of these cases varied considerably. Some were quite ill with temperatures of 102 to $103^{\circ} \mathrm{F}$., but not as severely ill as those in the septicaemic group. In others the general signs of inflammation were only slight with temperatures of 100 to $101^{\circ} \mathrm{F}$. Leucocyte counts varied from 7,000 to 25,000 and the sedimentation rate from 35 to $100 \mathrm{~mm}$. in the first hour (Westergren). The average duration of illness was four to five days (two to 10 days). Blood cultures were positive in two cases.

The local symptoms and signs depended upon the stage of the local lesion and although always definite, were sometimes difficult to detect because the neck of the femur is so deep seated and well padded by muscles. Not infrequently the diagnosis had at first been missed because of a lack of awareness of its possibility and failure to search for the tell-tale physical signs. 
TABLE 1

BACTERIOLOGY

\begin{tabular}{|c|c|c|c|}
\hline Organism & Sensitivity* & $\underset{\substack{\text { All } \\
\text { Cases } \\
\%)}}{\text {. }}$ & $\begin{array}{c}\text { Neck of } \\
\text { Femur } \\
\text { (24 Cases) }\end{array}$ \\
\hline $\begin{array}{l}\text { Mic-pvogenes } \\
\text { aureus }\end{array}$ & $\begin{array}{l}\text { All antibiotics } \\
\text { S.C.T.E.N. (not P.) } \\
\text { C. and E. only }\end{array}$ & $\begin{array}{r}81 \\
12 \\
4\end{array}$ & $\begin{array}{r}16(66 \%) \\
7(29 \%) \\
1 \quad(5 \%)\end{array}$ \\
\hline $\begin{array}{l}\text { Beta-haemolytic } \\
\text { Streptococcus }\end{array}$ & All antibiotics & $1 \cdot 5$ & - \\
\hline $\begin{array}{l}\text { Streptococcus } \\
\text { viridans }\end{array}$ & All antibiotics & 0.5 & - \\
\hline $\begin{array}{l}\text { Klebsiella } \\
\text { aerogenes }\end{array}$ & S.C.T.E.N. (not P.) & $0 \cdot 5$ & - \\
\hline Proteus $S p$ & S. and C. only & 0.5 & - \\
\hline
\end{tabular}

$$
\begin{aligned}
* \mathbf{S}=\text { Streptomycin } & \mathbf{E}=\text { Erythromycin } \\
\mathbf{C}=\text { Chloramphenicol } & \mathbf{N}=\text { Novobiocin } \\
\mathbf{T}=\text { Tetracycline } & \mathbf{P}=\text { Penicillin }
\end{aligned}
$$

Pain in the region of the affected hip and a limp or inability to use the limb were constant and present at all stages of the disease. During the early stage of acute inflammation, before the formation of pus, the only objective signs were pain on passive movement especially internal rotation and extension, and tenderness over the neck of the femur. The latter sign is detected by deep palpation between the ischial tuberosity and greater trochanter of the femur (Fig. 4). Two of the patients in this group were first seen at this stage and the duration of their illness was two days and four days. On the other hand there were three cases with histories of only two to three days where the disease was already at a more advanced stage. It is thus obvious that although the duration is an important factor, time alone cannot be the only guide to the stage of the disease.

Our experience has led us to believe that any signs in addition to local pain and tenderness indicate the presence of pus. When there is intraosseous pus formation, oedema is usually the first clinical indication: if the bone is superficial this is manifest as pitting oedema, but if the bone is

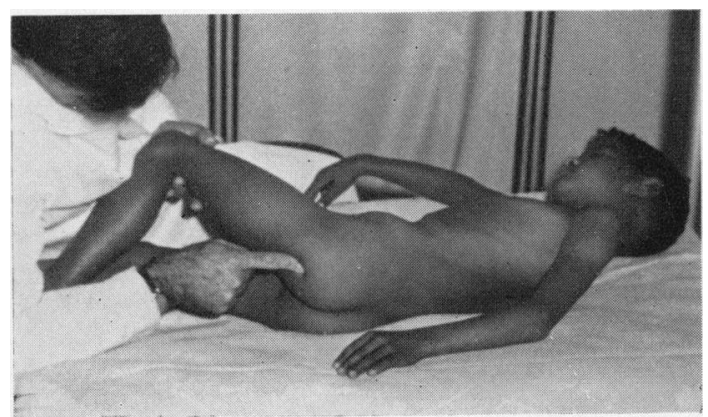

Fig. 4.-An early and constant sign in osteomyelitis of the femoral neck is tenderness on deep palpation between the ischial tuberosity and greater trochanter.

\begin{tabular}{|c|c|c|c|c|}
\hline \multicolumn{2}{|c|}{ Presentation } & Cases & $\begin{array}{l}\text { Duration of } \\
\text { Illness } \\
\text { (days) }\end{array}$ & $\begin{array}{c}\text { Positive } \\
\text { Blood } \\
\text { Culture }\end{array}$ \\
\hline $\begin{array}{c}\text { Bacteraemia and } \\
\text { tory fever .. }\end{array}$ & $\begin{array}{c}\text { nflamma- } \\
\ldots\end{array}$ & 11 & $\begin{array}{c}4-5 \\
(2-10)\end{array}$ & 2 \\
\hline Septicaemia .. & . & 12 & $\begin{array}{l}2 \cdot 5 \\
(1-4)\end{array}$ & 10 \\
\hline Severe toxaemia & $\cdots$ & $\begin{array}{c}4 \\
\text { (5 bones) }\end{array}$ & $\stackrel{9}{(6-18)}$ & 1 \\
\hline Totals & . & $\begin{array}{l}27 \\
\text { (28 hones) }\end{array}$ & $\begin{array}{c}6 \\
(1-18)\end{array}$ & 13 \\
\hline
\end{tabular}

TABLE 2

CLINICAL PRESENTATION

\begin{tabular}{|c|c|c|c|c|}
\hline Presentation & $\begin{array}{l}\text { No. } \\
\text { Drilled }\end{array}$ & \multicolumn{2}{|r|}{ Pus Found } & $\begin{array}{l}\text { Positive } \\
\text { Culture }\end{array}$ \\
\hline $\begin{array}{r}\text { Bacteraemia } \\
\text { (11 bones) }\end{array}$ & 11 & $\begin{array}{r}9-6 \\
3\end{array}$ & $\begin{array}{l}\text { intra-osseous only } \\
\text { intra- and extra- } \\
\text { osseous }\end{array}$ & 7 \\
\hline $\begin{array}{l}\text { Septicaemia } \\
\quad \text { (11 bones) }\end{array}$ & 11 & $\begin{array}{r}11-5 \\
6\end{array}$ & $\begin{array}{l}\text { intra-osseous only } \\
\text { intra- and extra- } \\
\text { osseous }\end{array}$ & 8 \\
\hline \multirow[t]{2}{*}{$\begin{array}{l}\text { Toxaemia } \\
\text { ( } 5 \text { bones })\end{array}$} & 5 & & $\begin{array}{l}\text { all intra- and extra- } \\
\text { osseous }\end{array}$ & 3 \\
\hline & 27 & \multicolumn{2}{|c|}{$\begin{array}{c}25-11 \text { intra-osseous only } \\
14 \text { intra- and extra- } \\
\text { osseous }\end{array}$} & 18 \\
\hline
\end{tabular}

TABLE 3

FORMATION OF PUS (27 BONES)

deeply situated there is only vague swelling. In osteitis of the femoral neck this important physical sign is often not obvious until a somewhat later stage, but will be detected earlier by measurement and assessment of the thickness of the skin (Fig. 5). Although intra-osseous pus was present in the remaining nine cases in this group (Table 3 ), swelling could not be detected in two of them. In these two cases the presence of pus was diagnosed

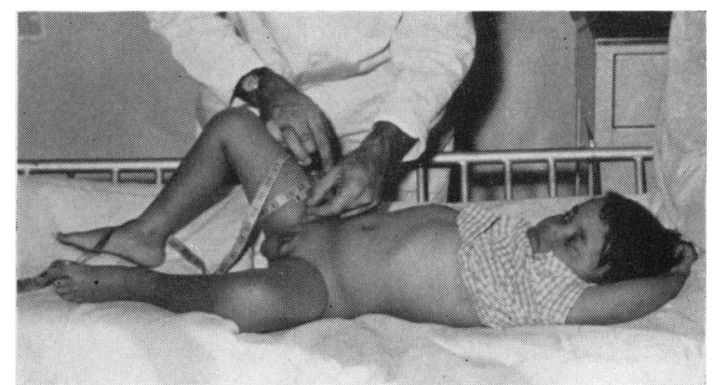

FIG. 5.-Slight swelling is detected by measurement and assessment of the thickness of the skin. 


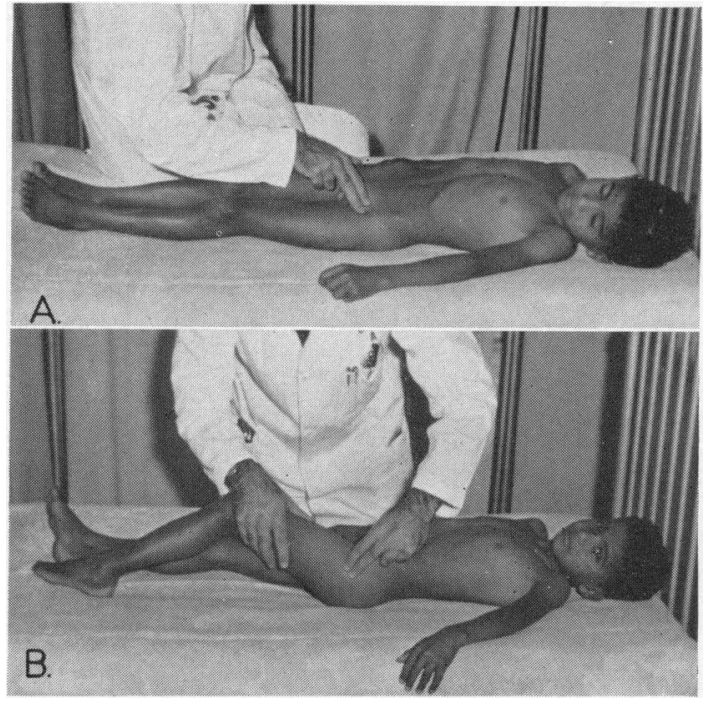

Fig. 6.- In more advanced cases the tenderness and swelling are present over Scarpa's triangle (6a), and the upper femur (6b).

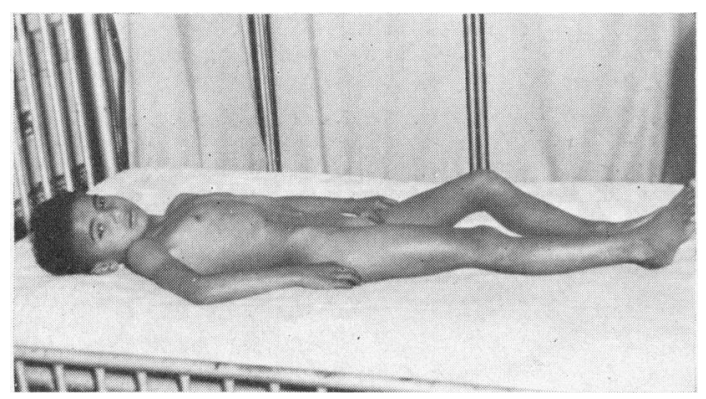

Fig. 7.-The hip is held in slight flexion, abduction and external rotation.

by assessing the response to conservative treatment as recommended by Trueta and Morgan (1954). The extent and intensity of the bone tenderness were accurately determined and recorded. The patients were then put on intensive antibiotic therapy. After 12 hours and 24 hours the same surgeon re-examined the patient. An increase in tenderness after 12 hours in one case and no marked decrease after 24 hours in the other, led to the correct diagnosis of intra-osseous pus formation.

Other signs of pus formation are local heat, redness and dilated veins. These are also more commonly seen in superficial bones and prompted Rutherford Morison's (Morison and Saint, 1935) aphorism: 'Cellulitis in a young limb means osteitis'. In the case of the femoral neck, however, these signs occur late and the diagnosis of pus formation should be made before they appear.

Once pus has extended through the bone into the subperiosteal space, local swelling, heat and redness are usually obvious, even in deep-seated bones. Extra-osseous as well as intra-osseous pus was present in three of our cases in this group (Table 3). In all of them there was visible swelling of the upper thigh, especially on the medial side and over Scarpa's triangle (Fig. 6a), extension of tenderness to the greater trochanter and upper femur (Fig. 6b) and increased muscle spasm which held the hip joint in some flexion, external rotation and abduction (Fig. 7). It should be noted that the duration of the illness in these cases varied from four to nine days and that in all of them the bone still contained pus under tension.

(2) Septicaemia-12 Cases. These children were all severely ill with fever of 103 to $107^{\circ} \mathrm{F}$., tachycardia of 140 to 160 , rigors or convulsions and often headache, drowsiness, delirium and epistaxis. In addition, symptoms such as flitting arthralgia, meningism, erythematous rashes, purpura, vomiting and diarrhoea tended to obscure the true diagnosis. Indeed, in two of the cases the patients also developed other lesions, namely pleurisy and pericarditis in one, and meningitis and pneumonia in the other.

In all the cases, the illness was of short duration (one to four days, with an average of $2 \cdot 5$ ), the leucocyte count was over $20,000(22,000$ to 45,000$)$ and the sedimentation rate was over $80 \mathrm{~mm}$. in the first hour $(85$ to 120$)$. Blood cultures were positive in 10 cases; the two cases with negative cultures had received antibiotic therapy before admission.

The local signs were often not obvious, although intra-osseous pus was present in 11 of the 12 cases (Table 3). These severely ill patients do not complain of the excruciating local pain usually found in others, and the local lesion may be missed unless constantly kept in mind and specifically sought for. In six of the cases local tenderness over the neck of the femur was the only positive evidence of osteomyelitis and in five of them the presence of pus was diagnosed because the tenderness increased during the first 12 to 24 hours of antibiotic therapy. The sixth improved and was not operated upon. In the remaining six cases there was also evidence of swelling, local heat, etc., indicative of extraosseous pus (Table 3), but in none were these signs very striking.

Not only are the local signs completely overshadowed by the general condition of the patient, but it is this type of case that tends to have multiple osseous lesions. Five of our patients had other 
bones involved, namely tibia (two cases), opposite femoral neck, lower femur and ulna. In such cases the lesion in the deep-seated femoral neck is easily missed, and was in fact at first overlooked in two of our cases.

(3) Severe Toxaemia-Four Cases (Five Bones). All these patients had a long history (six to 18 daysaverage nine days). They were severely toxic with temperatures of 102 to $105^{\circ} \mathrm{F}$. Leucocyte counts ranged from 18,000 to 63,000 , and the sedimentation rate from 50 to 95 . Blood culture was positive in one case only.

Local tenderness and swelling were well marked and extensive in all the cases. In two the swelling involved the whole thigh and fluctuation, indicative of pus in soft tissues, could be detected. Local heat, redness and dilated veins were equally prominent, and in two of the patients movements of the hip joint were markedly restricted, suggesting infection of the joint. In all these cases pus was found outside as well as inside the bone (Table 3), and in two of them the pus within the bone was still under tension.

\section{Diagnosis}

We did not accept the diagnosis of acute osteomyelitis as proven until one or both of the following criteria had been satisfied:

1. Pus in the bone as found at operation and confirmed by culture.

2. Radiological evidence of serial changes in the affected bone consistent with the presence of pyogenic infection.

Intra-osseous Pus. The femoral neck was explored in 26 cases ( 27 bones) and intra-osseous pus was found in 24 ( 25 bones, Table 3 ). In seven of these the pus was sterile on culture but all had received massive antibiotic therapy for long periods. On the other hand positive cultures were obtained in eight patients who had been on antibiotics for periods of three to 10 days.

In two cases only thin marrow fluid escaped and failed to reveal any growth on culture. Both presented with local pain and general inflammatory fever and on examination local tenderness was the only positive finding (Tables 2 and 3 ).

One patient who presented with septicaemia and minimal local signs which responded to conservative treatment, was not operated upon and it thus cannot be proven whether pus had yet been formed.

Radiological Changes. It is well known that radiological changes are conspicuous by their absence during the first 10 to 14 days of acute osteomyelitis.
Radiographs are, therefore, valueless in assisting early diagnosis. Indeed, if treatment can be started at a really early stage of the disease, it may prevent altogether the appearance of radiologically detectable bone changes (Trueta and Morgan, 1954). This, however, is rare in hospital practice (Trueta and Morgan, 1954), and, as far as we are aware, we have not had the good fortune to encounter such a case.

All our patients, including the case not operated upon, developed radiological changes after two to three weeks. The earliest signs were usually a small area of erosion in the neck of the femur and minimal periosteal reaction (Fig. 8). In later cases decalcification, gross destruction of the femoral neck and sequestrum formation (Fig. 9) could be seen. Grossly neglected cases proceeded to involvement of the hip joint and destruction of the femoral head, giving the appearance of so-called Tom Smith's

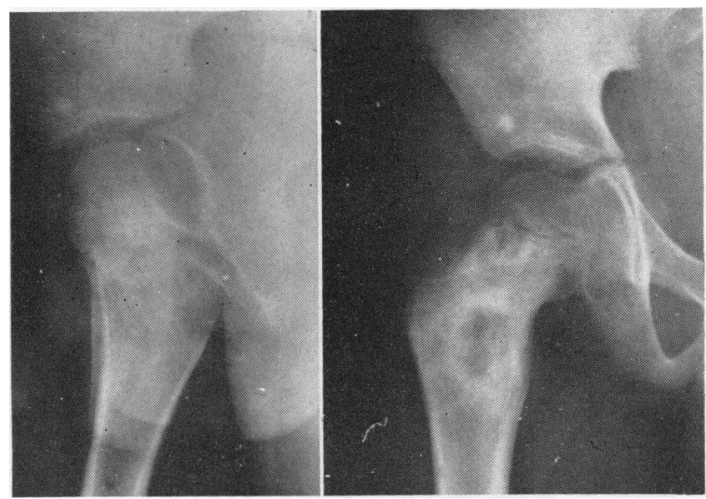

FIG. 8.-Early radiological changes: slight erosion of femoral neck and minimal periosteal reaction.

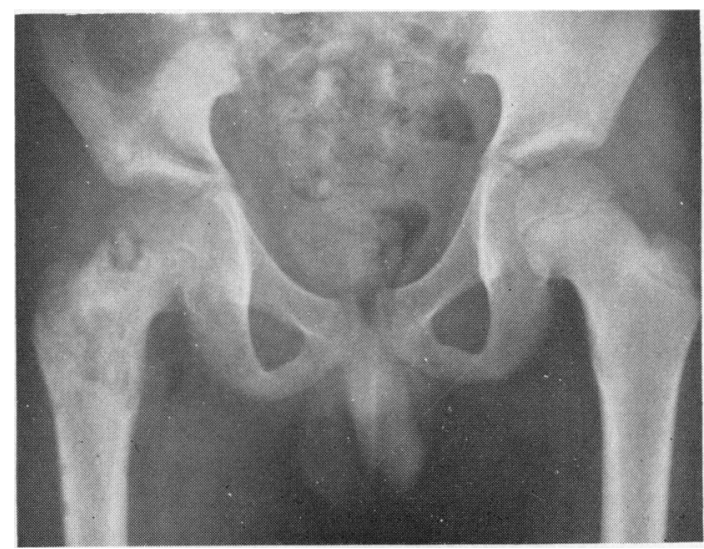

Fig. 9.-Extensive erosion and sequestrum formation. 


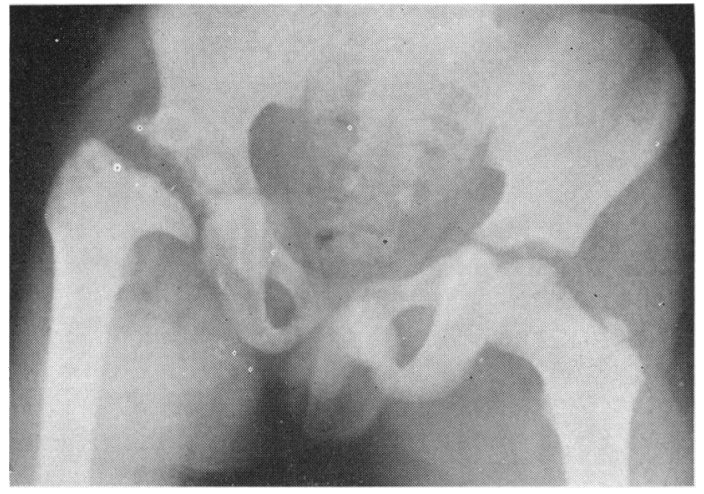

Fig. 10.-Gross destruction of femoral head and involvement of the hip joint: 'Tom Smith's arthritis'.

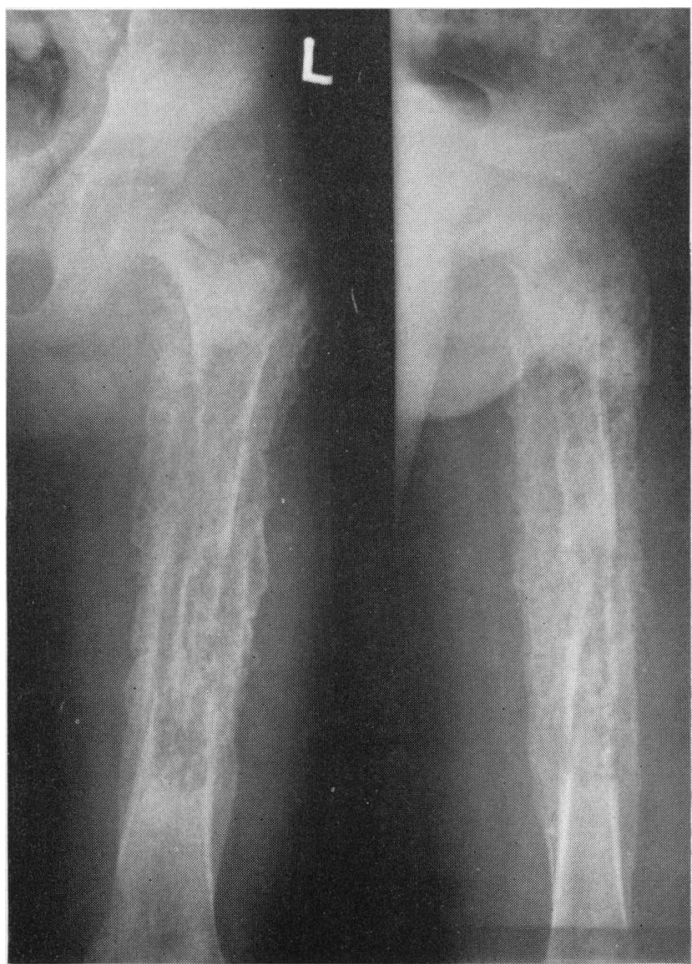

FIG. 11.-Involvement of the femoral shaft with extensive bone destruction and periosteal new bone formation.

arthritis (Fig. 10). (It is our belief that the majority of cases of Tom Smith's disease in patients over 2 years are in fact due to neglected osteomyelitis of the femoral neck.) In other late cases extension of the infection to the shaft of the femur may result in widespread destruction of bone, periosteal new bone formation and sequestration (Fig. 11).

\section{Treatment}

Our treatment of acute osteomyelitis is based on the following principles:

1. To combat the infection by adequate antibiotic therapy.

2. To provide rest.

3. To let out pus when it has formed.

4. To prevent complications.

Antibiotics. A great deal has been written on the value of antibacterial drugs in the treatment of acute osteomyelitis (Agerholm and Trueta, 1946; Higgins, Browne and Bodian, 1947; Dennison, 1952). The mortality of the disease has been reduced from about $25 \%$ without any drugs (Pyrah and Pain, 1933; McAdam, 1945; Higgins et al., 1947; Dennison, 1948 ) to $10 \%$ with sulphonamides (Dennison 1948) and to 2 to $3 \%$ with penicillin (Altemeier and Helmsworth, 1945; McAdam, 1945; Stevenson, 1946).

There are, however, certain reservations and limitations in connexion with antibiotic therapy which are often overlooked:

1. It has repeatedly been shown that antibiotics will cure the blood infection, but that progressive bone destruction continues despite amelioration of the general symptoms (Agerholm and Trueta, 1946; Dennison, 1952; Du Plessis, 1953; Trueta and Morgan, 1954). It is well known that antibiotics cannot sterilize an abscess (Agerholm and Trueta, 1946), and we have now had a large number of cases, including eight with osteitis of the femoral neck, in whom pus, which contained viable organisms, was evacuated from the bone after intensive and prolonged antibiotic therapy.

2. Antibiotic therapy, to be effective, must be correct, intensive and prolonged.

Correct antibiotic therapy obviously depends on culture of the organism from the blood or the bone, and the result of this is usually not available for two to three days. Much has been written on the efficacy of penicillin. We agree that it gives excellent results provided the organism is penicillin-sensitive and have used it in $80 \%$ of our cases (66\% of those with femoral neck involvement). However, in $20 \%$ of our cases the organisms have been insensitive to the drug and we, therefore, commence treatment with penicillin and one of the tetracycline group of drugs. (In desperately ill patients we prefer to use chloramphenicol or erythromycin and novobiocin because in $4 \%$ of our cases the organisms have been sensitive to these drugs only.) 


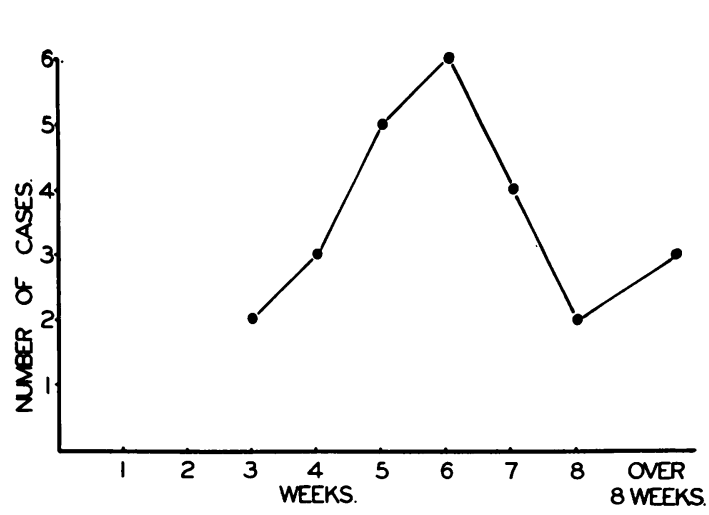

FIG. 12.-Duration of raised E.S.R. in 25 cases.

Massive dosage is particularly important as far as penicillin is concerned. We now give 2-3 million units per day depending on age, commencing with six-hourly injections. (This is changed to oral penicillin when the temperature subsides.) Tetracyclines are given in dosages of $15 \mathrm{mg}$. per $1 \mathrm{~b}$. per day.

Prolonged therapy is as important. Bitter experience has taught us that premature cessation of antibiotic treatment only too frequently leads to recrudescence or complications. We, therefore, continue not only until all general and local signs have completely subsided, but until the sedimentation rate has dropped below $20 \mathrm{~mm}$. in the first hour and remained there for at least a week. The duration of treatment in our cases has ranged from three to eight weeks (average six weeks) in uncomplicated cases (Fig. 12), and we feel most strongly that anything less is inadequate and may be disastrous. (In three complicated cases therapy was continued for three to six months.)

Rest. A century ago John Hilton (1863) said: 'Rest is the necessary antecedent to the healthy accomplishment of both repair and growth. This surely is the natural suggestion of a means towards an end which should never be lost sight of by the physician or surgeon.'

The value of immobilization in the treatment of acute osteomyelitis is often overlooked. It is true that immobilization is usually mentioned but its purpose seems to be 'to prevent fractures and dislocations' rather than to provide the rest referred to by John Hilton.

We have found that inadequate or too brief a period of immobilization results in recrudescence of infection, and predisposes to complications. It has, therefore, become our policy to immobilize the

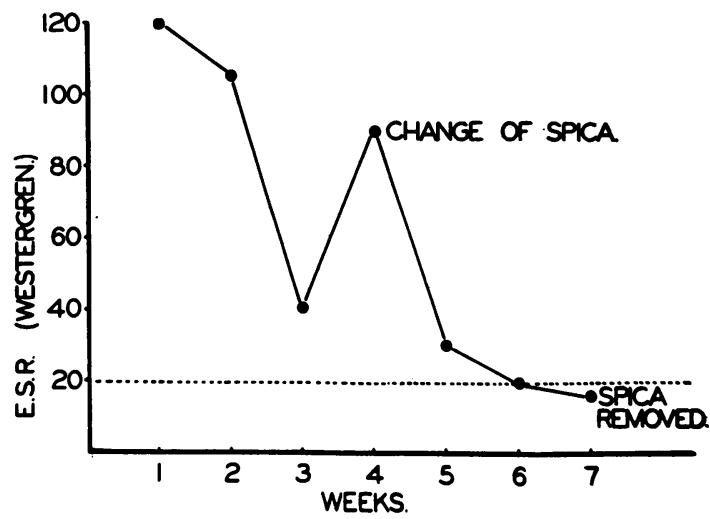

FIG. 13.-A typical charı of the weekly E.S.R. readings in an uncom plicated case. Note the effect of change of plaster.

bone in a plaster cast until the sedimentation rate has returned to normal. This index of activity has proved of great value (Fig. 12). First, it has been interesting to observe the effects of change of plaster, transport to a convalescent home and other movement on the sedimentation rate (Fig. 13). Second, in the past when we used to dispense with complete immobilization before the E.S.R. had returned to normal, our complication rate was almost twice as high as at present.

In the cases of osteitis of the femoral neck, this immobilization has been achieved by applying a plaster spica from waist to toes (Fig. 14). Plasters are changed at regular intervals and radiographs taken at the same time. Weekly sedimentation rates, leucocyte counts and haemoglobin estimations are carried out and recorded on a special chart. Complete immobilization is continued until clinically and radiologically there are no signs of activity and two successive E.S.R. readings are below $20 \mathrm{~mm}$. in the first hour. The average period of immobilization has been six weeks with a range of three to

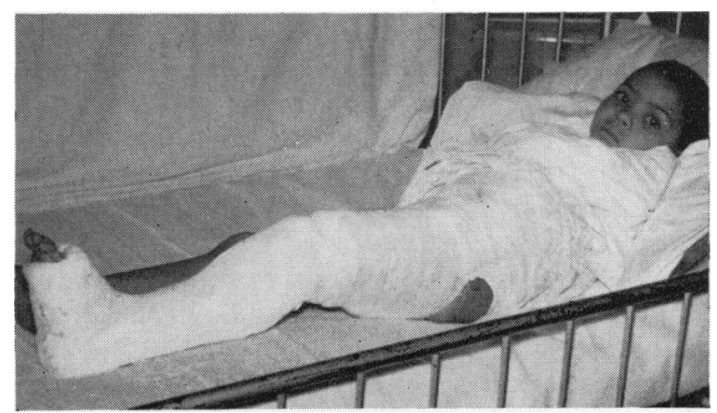

FIG. 14.-Adequate local rest is ensured by immobilization in a plaster spica from waist to toes. 


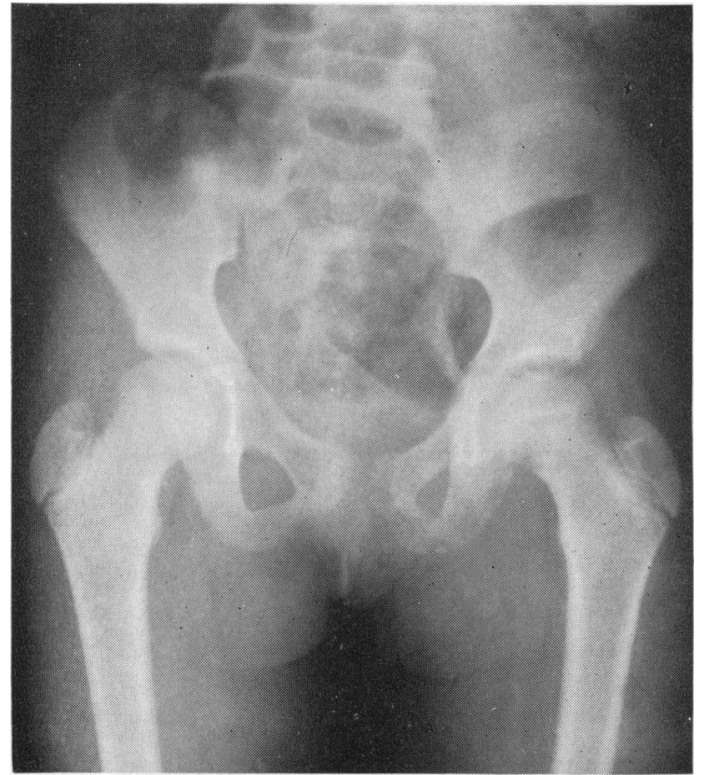

FIG. 15.-Osteomyelitis of the left pubic ramus. An initial, erroneous diagnosis of osteomyelitis of the femoral neck was made.

eight weeks in uncomplicated cases. (The average period of hospitalization for all cases of osteomyelitis has been 45 days.)

Drainage of Pus. Our policy follows that of Trueta and his colleagues (Agerholm and Trueta, 1946; Trueta and Morgan, 1954), and is based on the age-old dictum that 'where there is pus it should be let out' and the belief that adequate drainage of bone in patients over 2 years can be obtained only by an open operation and drilling.

Our criteria for the diagnosis of intra-osseous pus have already been mentioned and have been well tested by an experience which we regard as unparalleled. The diagnosis of osteitis of the neck of the femur, however, is often extraordinarily difficult and mistakes have been made. These include:

1. Drilling of the bone before pus has formed. This happened twice in 26 cases ( 27 bones), i.e. in $7 \cdot 4 \%$. In both cases, operated upon early in the series, the local physical signs were merely tenderness and restricted movement, but the bones were drilled immediately instead of awaiting the result of a 24-hour trial of antibiotic therapy. Although no pus was found, subsequent radiographs proved the diagnosis to be correct.

It is possible that these cases would have recovered without surgery. On the other hand the drilling does not disturb the architecture of the bone and in both cases marrow fluid escaped under tension. This tension is due to intramedullary oedema which tends to cut off the blood supply to adjacent areas and leads to thrombosis (Agerholm and Trueta, 1946). It may, therefore, be argued that the decompression was actually beneficial and allowed the circulating penicillin to reach the site of the disease. Both patients have done very well, with complete bone healing and restoration of full function.

2. Drilling of the femoral neck because of mistaken diagnosis. This happened twice (the cases are obviously not included in the series). In one child, after a negative exploration, the temperature, leucocytosis and sedimentation rate returned to normal within three days, and the patient was discharged after two weeks, perfectly fit. Subsequent radiographs revealed no bony changes and the correct diagnosis, in retrospect, was probably cellulitis. Another child, who also had a negative exploration, subsequently developed signs of osteitis of the pubic ramus (Fig. 15). In neither did the drilling procedure have any harmful effects.

It has been suggested that drilling is not necessary when pus has appeared under the periosteum. We cannot agree with this. Extra-osseous pus was present in 14 of our cases and in all of them pus was also found within the bone (Table 3). Indeed, in nine the intra-osseous pus was still under tension. This is in keeping with our overall experience, namely that the mere presence of pus outside the bone is not indicative of adequate decompression and that drilling is, therefore, necessary even when subperiosteal pus is found.

Our method consists of operating on the bone under general anaesthesia as soon as the patient is fit for surgery. In moderately ill patients who present with merely the usual general reaction to inflammation a few hours are profitably spent in rehydrating the patient and obtaining an adequate blood level of antibiotics. A delay of more than six to 12 hours is not regarded as justifiable, unless there is doubt about pus formation. If there is septicaemia it must first be overcome by intensive antibiotic therapy for 12 to 24 hours before surgery is undertaken. In neglected cases suffering from severe toxaemia due to the presence of a large abscess of long standing only slight improvement can be expected with antibiotic therapy, rehydration and blood transfusion and it is wise to operate as soon as the patient is considered fit for simple drainage of the abscess.

In the usual metaphyseal infection of long bones the standard approach is used to expose at least half the shaft of the affected bone which is then drilled in several places. In the case of the femoral 


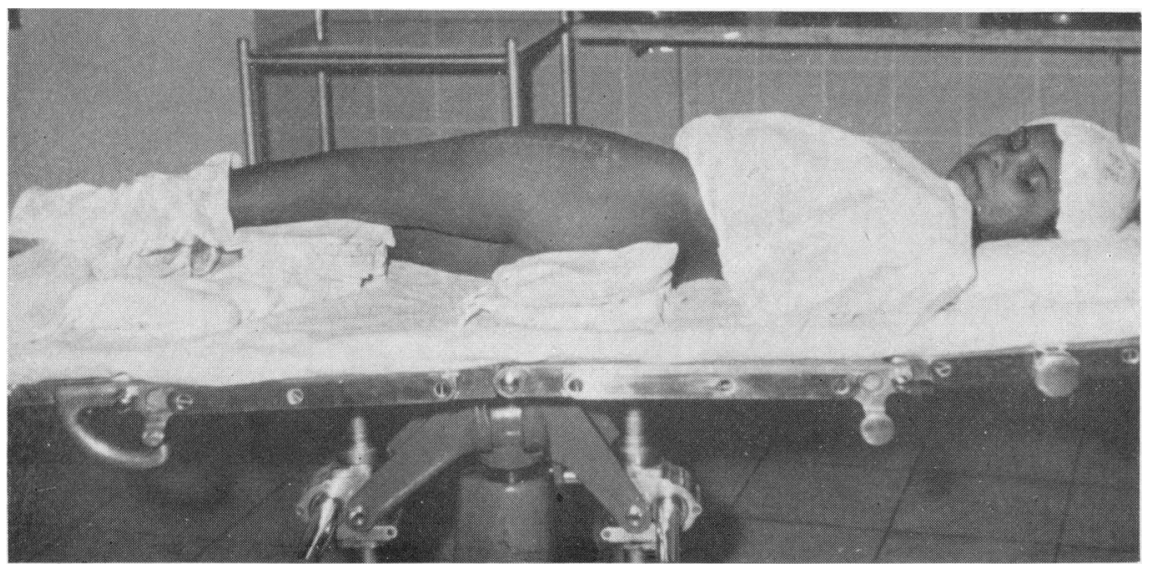

FIG. 16.-For drilling of the femoral neck the child is placed in the lateral position with a 45 degree backward tilt. Note the position of the scar.

neck the child is placed in the lateral position with the diseased side uppermost and with a 45-degree backward tilt (Fig. 16). When involvement of the hip joint is suspected, the joint is aspirated, using the lateral route. (The synovial fluid was turbid in two cases of this series, but in neither was pus obtained nor any growth on culture.)

The upper femoral shaft is approached through a lateral incision centred opposite the base of the greater trochanter (Fig. 16). Fascia lata, muscles and periosteum are incised and the periosteum elevated only just sufficiently to expose the bone. Subperiosteal pus, if present, is evacuated and a specimen taken for culture.

A small hole is drilled through the cortex of the femur at a point about $0.5 \mathrm{~cm}$. below the base of the greater trochanter. A hand awl is passed up the middle of the neck of the femur, bearing in mind the angle of the neck to the shaft and its anterior rotation on the shaft (Fig. 17). The femoral neck is very short in children and since it is inadvisable to traverse the epiphyseal plate because of the risk of infecting the head of the femur and hip joint, the awl is inserted for not more than 1-1.5 in. Radiographic control is of great value and has been used as a routine; an antero-posterior radiograph usually suffices because the cortex of the superior and inferior limits of the femoral neck can be felt by gentle manipulation of the awl. In young children the awl will suffice to decompress the neck, but in older children the hole has to be enlarged with a $\frac{3}{16}$-in. drill. Pus usually escapes from the drill hole with some force and a specimen is taken for culture.

The upper shaft of the femur is drilled as well in case the infection has already spread. (Pus was

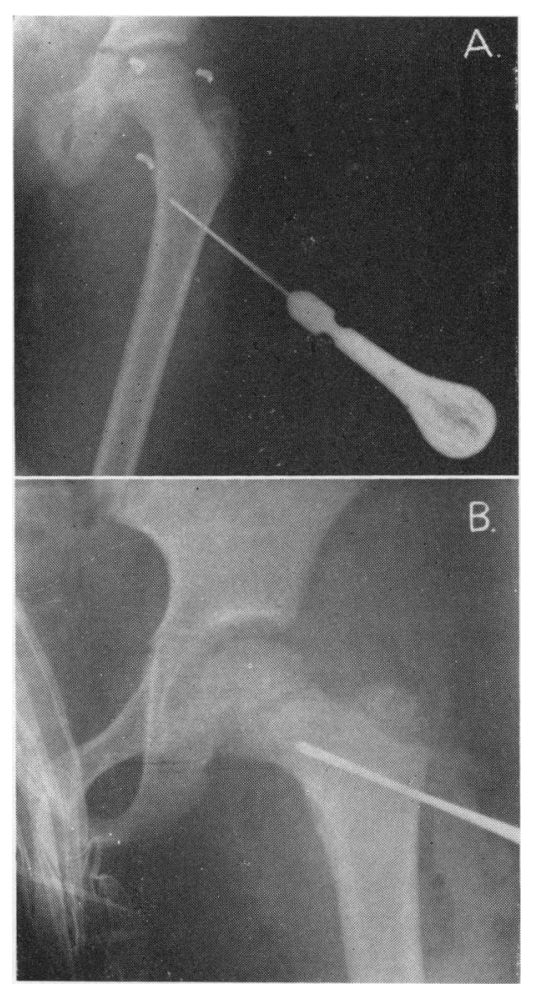

Fig. 17.-Radiographic control of decompression of the femoral neck. In $(a)$ the direction of the awl is obviously incorrect, but this is easily adjusted by paying attention to the three skin clips and 'aiming' at the middle clip. In (b) the drill is correctly sited.

found in six of our cases.) Drill holes are made at $1 \mathrm{~cm}$. intervals until at least two fail to yield any pus (Fig. 18). 


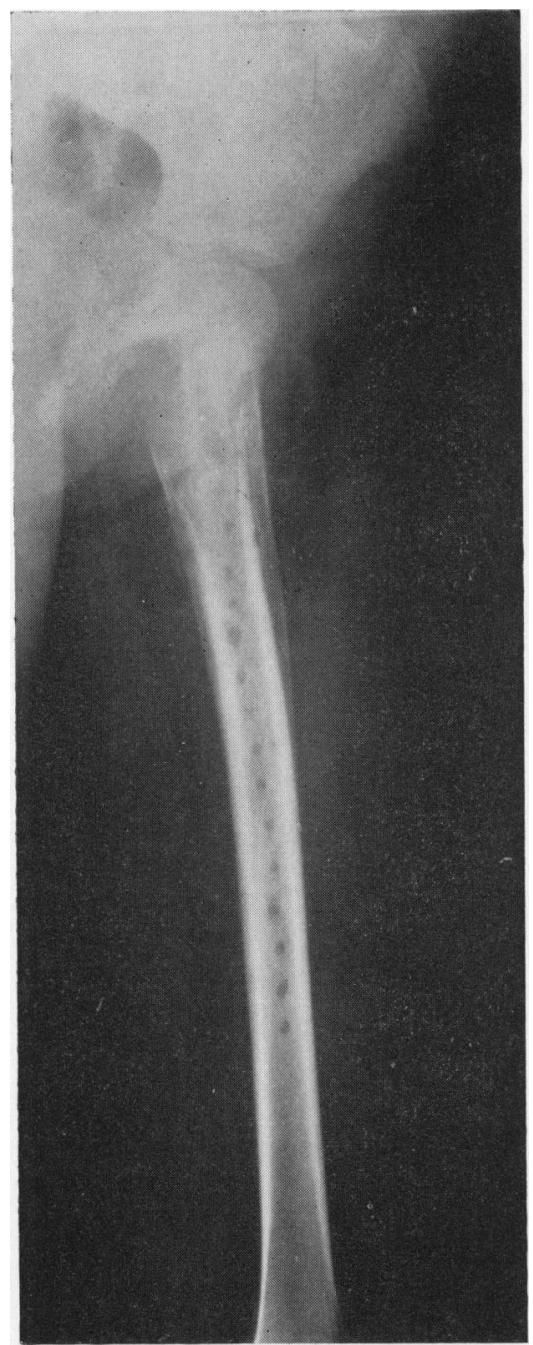

Fig. 18.-In this patient pus was found in the upper femoral shaft as well as in the neck of the femur. Note that the drill holes extend half-way down the shaft.
A corrugated rubber drain is inserted down to the bone and the wound is sutured. A plaster of paris spica is then applied from waist to toes (Fig. 14). On the fifth postoperative day a window is cut in the plaster over the wound under strictly aseptic conditions and the drain is removed. The window is replaced and securely closed and on the tenth day the sutures are similarly removed.

Prevention of Complications. When we are satisfied that all activity has ceased and there are no complications, antibiotic therapy and complete immobilization are discontinued and radiographs of the bone are taken. In some of the younger children who received treatment at an early stage the bones were sufficiently well calcified to render further protection unnecessary. In the majority including all those in the older age groups, there was still decalcification, and these patients were fitted with weight relieving calipers to prevent pathological fractures. They were followed up as out-patients and the calipers were removed when successive radiographs indicated adequate recalcification. Although the calipers, which were left on for an average period of six weeks, did not always achieve their original purpose, they did effectively limit the children's activity and prevent complications.

\section{Results}

The results of treatment are set out in Table 4 .

Early. There have been no deaths. (Our overall mortality for acute osteomyelitis has been $1.5 \%$, i.e. five deaths in 331 cases.)

Two patients (one with involvement of both femoral necks) are still under treatment and although both are doing well, it is still too early to assess the early results.

Of the remaining 25 cases, 24 were treated by medullary decompression. Three of these developed

TABLE 4

RESULTS OF TREATMENT

\begin{tabular}{|c|c|c|c|c|c|}
\hline & & $\begin{array}{c}\text { Deaths } \\
\text { (All Forms } \\
\text { of Treatment) }\end{array}$ & \multicolumn{2}{|c|}{$\begin{array}{l}\text { Early Results } \\
\text { (Drilling Only) }\end{array}$} & $\begin{array}{l}\text { Late Results (2-7 yrs) } \\
\text { (Drilling Only) }\end{array}$ \\
\hline Osteitis of neck of femur (27 cases)* & $\cdots$ & - & $\begin{array}{l}\text { Good } \\
\text { Complicated }\end{array}$ & $\begin{array}{r}21(87 \cdot 5 \%) \\
3(12 \cdot 5 \%)\end{array}$ & $\begin{array}{lr}\text { Full recovery } & 14(82.5 \%) \\
\text { Unsatisfactory } & 3(17.5 \%)\end{array}$ \\
\hline Osteitis in general (331 cases) & $\cdots$ & $5(1 \cdot 5 \%)$ & $\begin{array}{l}\text { Good } \\
\text { Complicated }\end{array}$ & $\begin{array}{r}178(88 \cdot 2 \%) \\
24(11 \cdot 8 \%)\end{array}$ & $\begin{array}{l}\text { Full recovery } 94(86.2 \%) \\
\text { Unsatisfactory } 15(13.8 \%)\end{array}$ \\
\hline
\end{tabular}

* One case treated conservatively: uncomplicated early but unsatisfactory late result. Two cases still under treatment. 
immediate complications. Two had been ill for more than a week (eight and 10 days) and were profoundly toxic on admission. At operation large extra-osseous abscesses were found as well as intraosseous pus under tension. Both developed septic arthritis of the hip joint after admission and required active treatment for six months; one also developed osteitis of the ulna. In the third patient, who had been ill for four days, surgery was delayed for three more days because of an error in diagnosis (the initial diagnosis was osteitis of the ilium). His illness was complicated by a protracted pyrexia for four weeks and the development of a sequestrum which gradually resorbed. $\mathrm{He}$ was discharged from hospital after three months with some limitation of movement of the hip joint.

In 22 cases the immediate results were excellent (21 were operated upon and one was treated conservatively). None developed metastatic foci, joint involvement, pathological fractures or dislocations, sequestra or recurrence after apparent recovery. Temperatures returned to normal after an average period of five days (one to 14 days). All the wounds healed by primary intention. The sedimentation rates reverted to normal after three to eight weeks (average six weeks) and a normal range of movement was re-established in all.

In summary, therefore, complications occurred in $12.5 \%$ of the surgically treated cases. This corresponds with our overall experience in cases treated by medullary decompression. In 202 cases of acute osteomyelitis of various bones so treated $11 \cdot 8 \%$ developed complications (Shandling, 1960). These results compare favourably with those of others who adopt a similar line of treatment (Agerholm and Trueta, 1946; Dennison, 1952; Du Plessis, 1953; Trueta and Morgan, 1954). It should be mentioned that in the past, before we inserted drains as a routine after drilling, early wound complications, such as serous collections and temporary sinuses, were twice as common as at present. In this connexion it should be noted that in Trueta and Morgan's (1954) series $50 \%$ of the patients developed a temporary sinus which 'occasionally appeared some time after the stitches had been removed'.

In contrast to these results, we found that in 67 of our cases who were treated entirely conservatively or by simple drainage of extra-osseous pus, early complications occurred in $35 \%$. This is in keeping with the findings of some (Agerholm and Trueta, 1946; Dennison, 1952), although at variance with those of others (Caldwell and Wickstrom, 1950; Altemeier and Largen, 1952).

Late. Eighteen of the patients have been followed up for seven years to two years. Among them are included the three patients who had early complications. Two of these have developed subluxation of the hip joint with marked limitation of movement, gross irregularity and thickening of the neck and shaft of the femur and considerable shortening. These two results are obviously bad, but there has been no recent recrudescence nor sequestrum formation. The third patient (who developed a sequestrum that resorbed) has slight limitation of movement of the hip joint, shortening of the limb and, on radiograph, widening, irregularity and patchy destruction of the neck and upper shaft of the femur.

A fourth case, ironically the patient who was treated conservatively, also has an unsatisfactory result. Clinically there is a slight limp and minimal limitation of hip joint movement, but the radiographs show patchy destruction of the neck and upper shaft of the femur.

In the remaining 14 cases $(82.5 \%$ of those treated surgically) the results have been excellent. All the children have returned to normal activity with full restoration of hip joint function, complete bone healing and non-adherent scars (Fig. 19). Increased growth in length and width of the femur is present in six cases (Fig. 20), but does not exceed $2 \mathrm{~cm}$. There have been no sinuses, no pathological fractures, no sequestra and no late recurrences.

These late results are somewhat less satisfactory than our overall results in acute osteomyelitis, in which $86 \cdot 2 \%$ of the cases followed up for one to seven years, have recovered completely with $100 \%$ normal function (Shandling, 1960), and not as good as those of Trueta and Morgan (1954) who obtained full function in $94 \%$ of their cases. However, allowance must be made for the following three factors:

1. The age of the patients. Trueta and Morgan (1954) have found that the age of the patient has a well-marked effect on the end result of osteomyelitis. 'The structure of the bone needs several years to revert to its normal pattern and the intensity of this reversion is progressively lost in approaching the final stages of growth.' Since half our patients were aged 10 to 12 years, the age factor may account for the comparatively long periods (six weeks) of hospitalization. Furthermore, the ages of the patients who developed complications and imperfect healing were 9,10 and 12 years.

2. The lateness of the diagnosis. The average duration of illness in our cases of osteomyelitis of the femoral neck was six days. In the two cases with the worst results the duration on admission was eight days and 10 days. In a third case who 


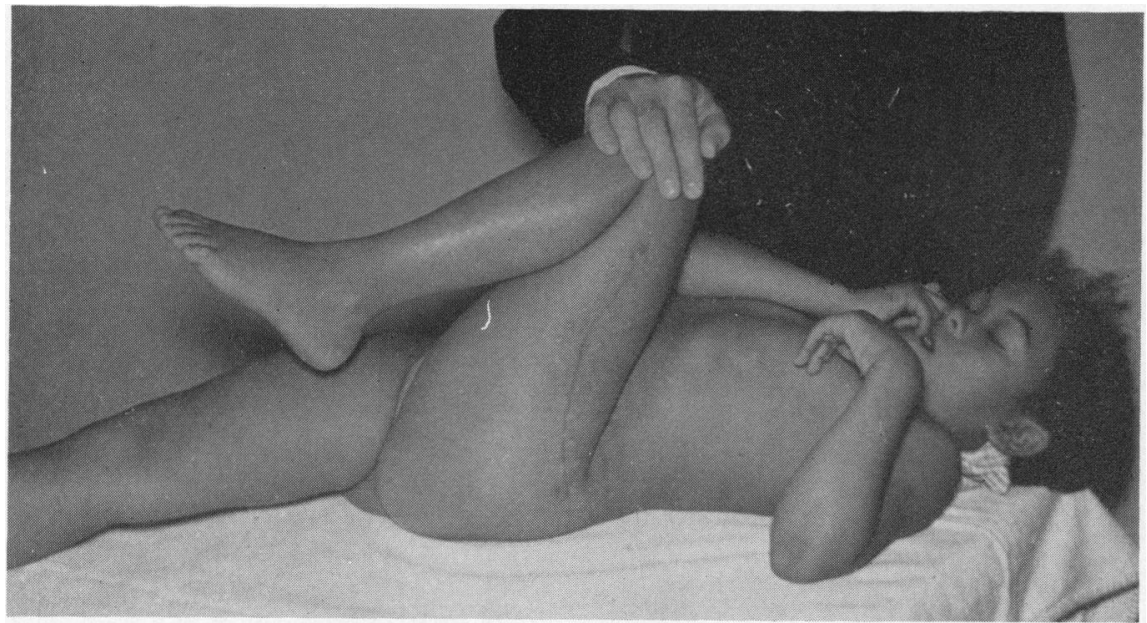

Fig. 19. - Late result in $82.5 \%$ of the cases, full function restored, scars well healed and non-adherent.

had been ill for four days, we were responsible for a further delay of three days before operating. (The fourth case with a poor result was treated conservatively and is not considered.)

Early diagnosis is undoubtedly one of the most important factors in the successful treatment of osteomyelitis and this may be excessively difficult in osteitis of the femoral neck. It is obviously a problem not only for the surgeon, but also for the

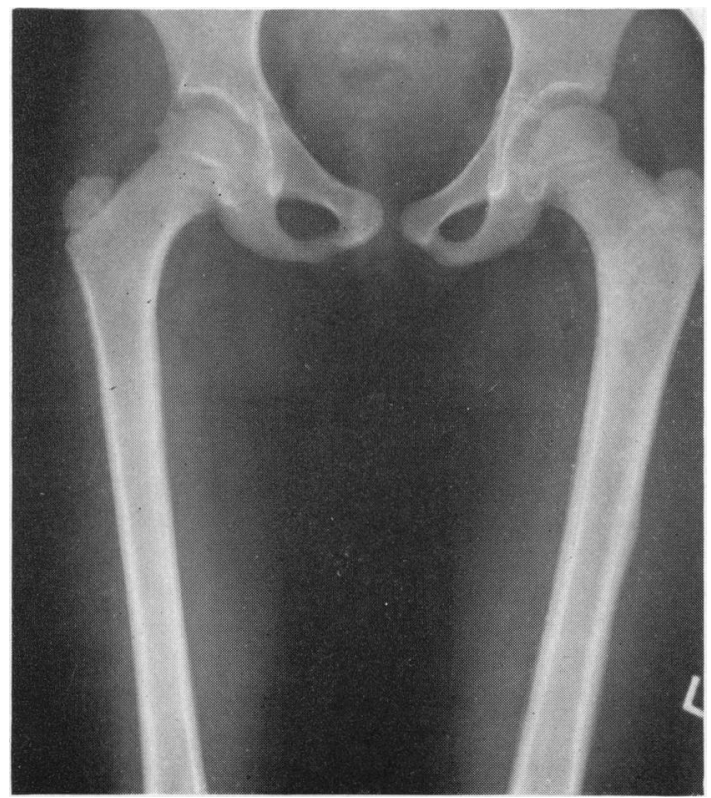

FIG. 20.-Late result, complete healing of the femur with slight increased growth in length and in width. general practitioner and paediatrician, and the disease must be given pride of place in the clinical consciousness of all of us.

3. The operations were performed by different surgeons (mainly registrars) and this unavoidably introduced a variable personal factor in the technique and thus also a small variation in the results.

\section{Conclusion}

In general our results compare favourably with those of others who have been treating acute osteomyelitis by medullary decompression (White and Dennison, 1952; Trueta and Morgan, 1954). Good results with conservative treatment have been claimed by some (Caldwell and Wickstrom, 1950; Altemeier and Largen, 1952), but these workers include in their series many cases without radiological signs of osteitis. Since these patients were not operated upon, the grounds for the diagnosis of osteomyelitis must be questioned, and the presence of bone infection cannot be accepted as proven. In our own experience and also in that of others (Agerholm and Trueta, 1946; Dennison, 1952), the results of conservative treatment leave much to be desired.

As far as osteomyelitis of the neck of the femur is concerned, too little has been written for comparison, but, judging from the numerous reports in the literature on septic arthritis of the hip joint with destruction of the head and neck of the femur, it is our impression that osteitis of the femoral neck has frequently been overlooked in the early stages only to be followed by this disastrous com- 
plication which is then regarded as the primary lesion.

While fully realizing that a good number of our cases have not yet reached the end of their evolution, we are convinced that the results of metaphyseal decompression are superior to those of conservative treatment, and feel justified in continuing our method of treatment, particularly in acute osteomyelitis of the neck of the femur. There is no room for complacency, however, and our objective should be $100 \%$ satisfactory results. To achieve this, early diagnosis, as always, is at a premium and can be made possible only if every practitioner, paediatrician and surgeon is constantly aware of the possibility of acute osteomyelitis and sufficiently alert to detect the early signs of the disease.

We would like to express our indebtedness to our colleagues and registrars who treated many of these patients; particularly to Mr. Arnold Katz who is in charge of our Osteitis Ward. Professor A. Kipps and Dr. D. McKenzie and their respective Bacteriological Departments have co-operated to the full at all times, and thanks are also due to the Medical Superintendents of the Red Cross War Memorial Children's Hospital and Groote Schuur Hospital for access to the case records. Finally, we wish to thank Mr. G. McManus, Miss R. E. Malan and Mrs. P. Kottler for their help with the photography and preparation of the manuscript.

\section{REFERENCES}

Agerholm, M. and Trueta, J. (1946). Acute haematogenous osteomyelitis treated with penicillin. Lancet, 1, 877.

Altemeier, W. A. and Helmsworth, J. A. (1945). Penicillin therapy in acute osteomyelitis. Surg. Gynec. Obstet., 81, 138.

- and Largen, T. (1952). Antibiotic and chemotherapeutic agents in infections of the skeletal system. J. Amer. med. Ass., $150,1462$.

Caldwell, G. A. and Wickstrom, J. (1950). The closed treatment of acute hematogenous osteomyelitis. Ann. Surg., 131, 734.

Dennison, W. M. (1948). Haematogenous osteitis in children Preliminary report on treatment with penicillin. $J$. Bone $J t$ Surg. 30B, 110 .

Surg., 30B, The penicillin treatment of acute haematogenous osteitis: A review of some unsatisfactory results. Glasg. med. $J$. $33,16$.

Du Plessis, D. J. (1953). Acute haematogenous osteomyelitis. S. Afr. med. J., 27, 584.

Higgins, T. T. Browne, D and Bodian, M. (1947). A penicillintreated series of cases of osteomyelitis in childhood. Brit. med. J., 1,757 .

Hilton, J. (1863). On the Influence of Mechanical and Physiological Rest. Bell and Daldy, London.

McAdam, I. W. J. (1945). Penicillin treatment of acute haematogenous osteomyelitis. Brit. J. Surg., 33, 167.

Mitchell, A. (1928). The treatment of acute osteomyelitis. Proc. roy. Soc. Med., 21, 1386.

Morison, R. and Saint, C. F. M. (1935). An Introduction to Surgery, 3rd ed. John Wright, Bristol.

Ober, F. R. (1938). Osteomyelitis in children. Amer. J. Surg. 39, 319.

Pyrah, L. N. and Pain, A. B. (1933). Acute infective osteomyelitis: A review of 262 cases. Brit. J. Surg., 20, 590.

Shandling, B. (1960). Acute haematogenous osteomyelitis: A review of 300 cases treated during 1952-1959. S. Afr. med.J., 34, 520 .

Stevenson, G. H. (1946). Some aspects of osteomyelitis. Glasg. med. $J ., 27,367$.

Trueta, J. and Morgan, J. D. (1954). Late results in the treatment of one hundred cases of acute haematogenous osteomyelitis. Brit. J. Surg. 41, 449

White, M. and Dennison, W. M. (1952). Acute haematogenous osteitis in childhood; a review of 212 cases. J. Bone Jt Surg., 34B, 608. 